\title{
Event-by-event distributions of azimuthal asymmetries in ultrarelativistic heavy-ion collisions
}

\author{
H. Niemi ${ }^{a}$, G. S. Denicol ${ }^{b, c}$, H. Holopainen ${ }^{d}$, and P. Huovinen ${ }^{c, d}$ \\ ${ }^{a}$ Department of Physics, P.O.Box 35, FI-40014 University of Jyväskylä, Finland \\ ${ }^{b}$ Department of Physics, McGill University, 3600 University Street, Montreal, Quebec, H3A 2T8, Canada \\ ${ }^{c}$ Institut für Theoretische Physik, Johann Wolfgang Goethe-Universität, \\ Max-von-Laue-Str. 1, D-60438 Frankfurt am Main, Germany and \\ ${ }^{d}$ Frankfurt Institute for Advanced Studies, Ruth-Moufang-Str. 1, D-60438 Frankfurt am Main, Germany
}

\begin{abstract}
Relativistic dissipative fluid dynamics is a common tool to describe the space-time evolution of the strongly interacting matter created in ultrarelativistic heavy-ion collisions. For a proper comparison to experimental data, fluid-dynamical calculations have to be performed on an event-by-event basis. Therefore, fluid dynamics should be able to reproduce, not only the event-averaged momentum anisotropies, $\left\langle v_{n}\right\rangle$, but also their distributions. In this paper, we investigate the event-by-event distributions of the initial-state and momentum anisotropies $\epsilon_{n}$ and $v_{n}$, and their correlations. We demonstrate that the event-by-event distributions of relative $v_{n}$ fluctuations are almost equal to the event-by-event distributions of corresponding $\epsilon_{n}$ fluctuations, allowing experimental determination of the relative anisotropy fluctuations of the initial state. Furthermore, the correlation $c\left(v_{2}, v_{4}\right)$ turns out to be sensitive to the viscosity of the fluid providing an additional constraint to the properties of the strongly interacting matter.
\end{abstract}

\section{INTRODUCTION}

Relativistic dissipative fluid dynamics is the most widely employed model to describe the space-time evolution of the quark-gluon plasma (QGP) created in ultrarelativistic heavy-ion collisions. It was the success of fluid-dynamical models in describing the large azimuthal momentum anisotropies observed in heavy-ion collisions that led to our current picture of the QGP, as a strongly interacting fluid with one of the smallest shear viscosity to entropy density ratios, $\eta / s$, ever observed [1].

The azimuthal momentum anisotropy is characterized in terms of the coefficients $v_{n}$ of the Fourier expansion of the single particle azimuthal distribution:

$$
\begin{aligned}
\frac{\mathrm{d} N}{\mathrm{~d} y \mathrm{~d} \phi} & =\frac{\mathrm{d} N}{\mathrm{~d} y}\left[1+2 v_{1} \cos \left(\phi-\psi_{1}\right)+2 v_{2} \cos \left[2\left(\phi-\psi_{2}\right)\right]+\ldots\right], \\
v_{n} & =\frac{\int \mathrm{d} \phi \cos \left[n\left(\phi-\psi_{n}\right)\right] \frac{\mathrm{d} N}{\mathrm{~d} y \mathrm{~d} \phi}}{\int \mathrm{d} \phi \frac{\mathrm{d} N}{\mathrm{~d} y \mathrm{~d} \phi}}=\left\langle\cos \left[n\left(\phi-\psi_{n}\right)\right]\right\rangle,
\end{aligned}
$$

where $\psi_{n}$ is the event-plane angle, $\psi_{n}=(1 / n) \arctan \left(\left\langle p_{T} \sin n \phi\right\rangle /\left\langle p_{T} \cos n \phi\right\rangle\right)$, and $\phi$ is the transverse momentum azimuthal angle. One of the main features of the fluid-dynamical description of the expansion is that the anisotropy originates from the azimuthal anisotropy of the initial density profile. In the literature this initial anisotropy is quantified in terms of coefficients $\epsilon_{m, n}$ :

$$
\epsilon_{m, n}=-\frac{\int \mathrm{d} x \mathrm{~d} y r^{m} \cos \left[n\left(\phi-\Psi_{m, n}\right)\right] \varepsilon\left(x, y, \tau_{0}\right)}{\int \mathrm{d} x \mathrm{~d} y r^{m} \varepsilon\left(x, y, \tau_{0}\right)},
$$

where $\varepsilon$ is the energy density, $r^{2}=x^{2}+y^{2}, \phi$ is now the spatial azimuthal angle, and $\Psi_{m, n}$ is the participant angle, defined as

$$
\Psi_{m, n}=\frac{1}{n} \arctan \frac{\int \mathrm{d} x \mathrm{~d} y r^{m} \sin (n \phi) \varepsilon\left(x, y, \tau_{0}\right)}{\int \mathrm{d} x \mathrm{~d} y r^{m} \cos (n \phi) \varepsilon\left(x, y, \tau_{0}\right)}+\pi / n .
$$

In the following we will concentrate on the anisotropies $\epsilon_{2, n}$, and use a shorthand $\epsilon_{n} \equiv \epsilon_{2, n}$.

In fluid-dynamical calculations, a linear relation between $v_{2}$ and $\epsilon_{2}$ was found, i.e., $v_{2} \propto \epsilon_{2}$ [2]. The proportionality coefficient was shown to depend, not only on the properties of the fluid such as the equation of state and viscosity, but also on the initial density, freeze-out temperature, and resonance content of the late hadronic state [2 4 ].

The initial conditions in these fluid dynamical calculations were always smooth, constructed as an average over infinitely many individual collisions of the particular centrality. It was thought that the use of this kind of averaged initial conditions would lead to a good description of observables which were averaged over many events. In other words, the anisotropy $v_{2}$ computed using the event-averaged initial condition was expected to be equal to the $\left\langle v_{2}\right\rangle_{\mathrm{ev}}$ 
observed in the collisions, where $\langle\ldots\rangle_{\mathrm{ev}}$ corresponds to an average over all the events of the corresponding centrality class.

Recently, it has been realized that, in order to obtain a proper comparison with experimental data, fluid-dynamical calculations have to be performed on an event-by-event basis. This was first pointed out by Kodama et al. [5] almost ten years ago. However, this view only become widely accepted years later, after the work of Alver and Roland [6]. They showed that $\epsilon_{3}$ and, consequently, $v_{3}$ are non-zero in a single event. This is in contrast to the traditionally used event-averaged initial conditions in fluid-dynamical models which had zero $\epsilon_{3}$ and $v_{3}$. Furthermore, Alver and Roland demonstrated that such finite value of $v_{3}$ can be observed in heavy-ion collisions. This finding made $\left\langle v_{3}\right\rangle_{\mathrm{ev}}$ as important observable as $\left\langle v_{2}\right\rangle_{\mathrm{ev}}$ for probing the properties of the dense matter formed in heavy-ion collisions, and led to several works studying the behavior of observables in an event-by-event fluid-dynamical description [7, 8].

On the other hand, if fluid dynamics can be applied to describe individual ultrarelativistic heavy-ion collisions, it must be able to describe $v_{n}$ in every collision, not only the average $\left\langle v_{n}\right\rangle_{\mathrm{ev}}$. Therefore it must be able to reproduce the distribution $\mathcal{P}\left(v_{n}\right)$ of $v_{n}$ in an ensemble of events too. To confirm the applicability of fluid dynamics to describe the expansion stage of heavy-ion collisions, it is thus not enough to check whether the event-averaged values of $v_{n}$ agree with the data, but one must also check whether their distributions, $\mathcal{P}\left(v_{n}\right)$, match what is experimentally observed. Recently, the distributions of $v_{2}, v_{3}$, and $v_{4}$ were measured at the LHC by the ATLAS collaboration [9]. Also, the first fluid-dynamical calculations of these distributions were performed by Gale et al. [10].

In this paper, we study the event-by-event probability distribution of the Fourier coefficients $v_{n}, P\left(v_{n}\right)$, and how they are correlated with the initial state anisotropies $\epsilon_{n}$ event-by-event. The goal of this paper is not to attempt a comparison with experimental data, but to explore how these distributions and correlations are affected by the fluid viscosity and initialization of the system. In this way, it will be possible to understand what can be learned by measuring such event-by-event distributions.

In the following we explain our fluid dynamical model in section [1] and show our results in section $\amalg$. Subsection IIIA is dedicated to an analysis of the event-by-event correlation between initial condition and flow anisotropy, while in subsections ЩIB and ЩIC we show our results for probability distributions of scaled anisotropy $\delta v_{n}, P\left(\delta v_{n}\right)$, and linear correlation coefficients $c\left(v_{n}, v_{m}\right)$, respectively. In section [V] we summarize our findings and make our conclusions.

\section{MODEL}

To generate the initial states event-by-event, we use a Monte-Carlo Glauber model as implemented in Ref. [8]. In this model, nucleons are distributed into nuclei according to Woods-Saxon distribution. NN-correlations and finite size effects are neglected since they have a negligible effect on the anisotropy coefficients [11]. In an event with a given impact parameter, nucleons from different nuclei are assumed to collide when their transverse distance $d$ is small enough, i.e., when $d^{2}<\sigma_{N N} / \pi$.

We consider two initial conditions, in which the initial entropy density, $s$, at $\tau_{0}=1 \mathrm{fm}$, is evaluated as

$$
s(x, y)=W \sum_{i=1}^{N_{\text {part, bin }}} \exp \left\{-\left[\left(x-x_{i}\right)^{2}+\left(y-y_{i}\right)^{2}\right] /\left(2 \sigma^{2}\right)\right\},
$$

where $x_{i}$ and $y_{i}$ are the spatial coordinates of either wounded nucleons (initial condition sWN) or binary collisions (initial condition sBC), given by the Monte-Carlo Glauber model. $W$ is a normalization constant fixed to provide the observed multiplicity and $\sigma=0.8 \mathrm{fm}$ is the spatial scale of a wounded nucleon or a binary collision. The centrality classes are determined according to the number of binary collisions (for initial condition sBC) or the number of participants (for initial condition sWN). The initial fluid velocity and shear-stress tensor are set to zero and we neglect the effects of bulk viscosity.

For the fluid-dynamical evolution, we use the model previously employed in Ref. [12]. We describe the time evolution of the fluid in the central rapidity region assuming boost invariance and a zero baryochemical potential. The equations of motion are given by the conservation laws for energy and momentum:

$$
\partial_{\mu} T^{\mu \nu}=0
$$

where $T^{\mu \nu}=(\varepsilon+p) u^{\mu} u^{\nu}-g^{\mu \nu} p+\pi^{\mu \nu}$, with $\varepsilon, p, u^{\mu}$, and $\pi^{\mu \nu}$ being the energy density, the thermodynamic pressure, the fluid 4-velocity, and the shear-stress tensor, respectively. We use the lattice QCD and hadron resonance gas based equation of state $s 95 p$-PCE-v1 [13] with chemical freeze-out at temperature $T_{c h e m}=150 \mathrm{MeV}$. The evolution equation of the shear-stress tensor is given by transient relativistic fluid dynamics [14, 15]:

$$
\Delta_{\alpha \beta}^{\mu \nu} \tau_{\pi} D \pi^{\alpha \beta}+\pi^{\mu \nu}=2 \eta \sigma^{\mu \nu}-\frac{4}{3} \pi^{\mu \nu} \theta-\frac{10}{7} \Delta_{\alpha \beta}^{\mu \nu} \sigma_{\lambda}^{\alpha} \pi^{\beta \lambda}+\frac{74}{315 \eta} \Delta_{\alpha \beta}^{\mu \nu} \pi_{\lambda}^{\alpha} \pi^{\beta \lambda}
$$


where $\eta$ is the shear viscosity coefficient, $D=u^{\mu} \partial_{\mu}$ is the co-moving time derivative, $\sigma^{\mu \nu}=\Delta_{\alpha \beta}^{\mu \nu} \partial^{\alpha} u^{\beta}$ is the shear tensor, $\theta=\partial_{\mu} u^{\mu}$ is the expansion rate, and $\Delta_{\alpha \beta}^{\mu \nu}=\left(\Delta_{\alpha}^{\mu} \Delta_{\beta}^{\nu}+\Delta_{\alpha}^{\nu} \Delta_{\beta}^{\mu}-2 / 3 \Delta^{\mu \nu} \Delta_{\alpha \beta}\right) / 2$, with $\Delta^{\mu \nu}=g^{\mu \nu}-u^{\mu} u^{\nu}$. The transport coefficients of the non-linear terms on the right-hand side of the Eq. 6 were taken in the massless limit, in the 14-moment approximation, and the relaxation time was assumed to be $\tau_{\pi}=5 \eta /(\varepsilon+P)$ [15, 16]. Here, we have not included the nonlinear terms related to the vorticity tensor. Note that the last two terms in Eq. (6) were not included in our previous studies [12]. While such terms can have a significant effect on many observables, they are not relevant for the results discussed in this paper. We shall leave a detailed investigation of the effect of such terms to a future work. The equations of motion were solved numerically using the SHASTA algorithm, whereas the evolution equations for shear stress (Eq. 6) were solved using simple finite differencing scheme. For more details see Refs. [12, 17].

The hadron spectra are calculated with the Cooper-Frye freeze-out procedure [18] using the decoupling temperature $T_{f}=100 \mathrm{MeV}$, which was shown to give reasonable agreement with both the $p_{T}$-spectrum and $\left\langle v_{2}\right\rangle_{\mathrm{ev}}$ for pions at RHIC when a temperature-dependent $\eta / s$ was used, see Refs. 12]. In this work, we use constant values of viscosity, $\eta / s=0$ and 0.16. Nevertheless, the $p_{T}$-spectrum and $\left\langle v_{2}\right\rangle_{\mathrm{ev}}$ remain close to what is actually observed at RHIC. Since our main purpose is not the comparison to experimental observables, we adjusted only the initial entropy density to fit the observed multiplicity, but kept all the other parameters unchanged. Finally, we use Israel and Stewart's 14-moment ansatz for the dissipative correction to the local equilibrium distribution function,

$$
\delta f_{i}=f_{0 i} \frac{p_{i}^{\mu} p_{i}^{\nu} \pi_{\mu \nu}}{T^{2}(\varepsilon+p)}
$$

where $f_{0 i}=\left\{\exp \left[\left(u_{\mu} p_{i}^{\mu}-\mu_{i}\right) / T\right] \pm 1\right\}^{-1}$ is the local equilibrium distribution function, with the index $i$ indicating different hadron species and $p_{i}^{\mu}$ the four-momentum of the corresponding hadron. After calculating the thermal spectra, we include the contribution from all 2- and 3-particle decays of unstable resonances up to $1.1 \mathrm{GeV}$ mass.

It should be noted that because we do not generate particle ensembles at any point we always know the direction of the event plane and the magnitude of $v_{n}$ exactly. Experimentally, one measures a finite number of particles, which smears the observed distribution of $v_{n}$. However, the final experimental result for the $v_{n}$ distributions undergoes an unfolding procedure that is supposed to remove such a smearing [9]. Therefore, for a comparison with data, one can use the particle distributions computed with fluid dynamics without generating an ensemble of particles. A more detailed way would be to generate the particle ensembles and apply the same complicated unfolding procedure used by the experimentalist to obtain the $v_{n}$ distribution, but this procedure would be an unnecessary complication for the purpose of this work.

\section{RESULTS}

In this work we consider $\mathrm{Au}+\mathrm{Au}$ collisions at $\sqrt{s_{N N}}=200 \mathrm{~A} \mathrm{GeV}$. All the results shown in this paper are for positively charged pions. For each centrality class a total of 2000 events were computed. The Fourier coefficients and the initial-state anisotropies were calculated according to Eqs. (11), and (2), respectively. In the following, we consider two constant values for the shear viscosity to entropy density ratio, $\eta / s=0$ and 0.16 .

\section{A. Correlations}

As mentioned in the Introduction, it has been known for a long time that the event averaged $v_{2}$, and the eccentricity of the averaged initial state, $\epsilon_{2}$ are approximately linearly related [2]. Similar relation has been found for $\epsilon_{3}$ and the average $v_{3}$ but not for $\epsilon_{4}$ and $v_{4}[19$, 20]. Here we study whether similar relations hold event-by-event by evaluating the linear correlation between the harmonics $v_{n}$ and $\epsilon_{n}$. We use the linear correlation coefficient,

$$
c(a, b)=\left\langle\frac{\left(a-\langle a\rangle_{\mathrm{ev}}\right)\left(b-\langle b\rangle_{\mathrm{ev}}\right)}{\sigma_{a} \sigma_{b}}\right\rangle_{\mathrm{ev}}
$$

where $\sigma_{a}$ is the standard deviation of the quantity $a$. This correlation function is $1(-1)$ if $a$ and $b$ are linearly (anti-linearly) correlated and zero in the absence of linear correlation.

The 2-dimensional histograms in Figs. 13 show the correlations between $\epsilon_{2}$ and $v_{2}, \epsilon_{3}$ and $v_{3}$, and $\epsilon_{4}$ and $v_{4}$, respectively, for the $20-30 \%$ centrality class. To study the effect of both viscosity and initialization on these correlations, we show the correlations in three different cases: (a) sBC initialization with $\eta / s=0$, (b) sBC initialization with $\eta / s=0.16$, and (c) sWN initialization with $\eta / s=0.16$. 

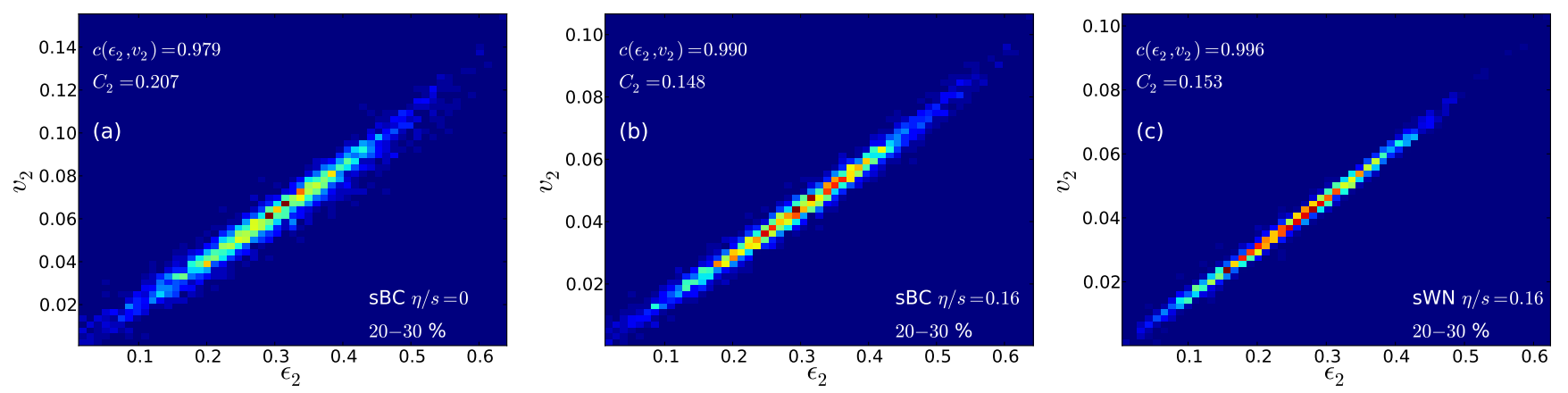

FIG. 1. $\epsilon_{2}$ and $v_{2}$ of pions in the $20-30 \%$ centrality class using different initializations and viscosities. a) $\mathrm{sBC}$ and $\eta / s=0$, b) $\mathrm{sBC}$ and $\eta / s=0.16$ and c) $\mathrm{sWN}$ and $\eta / s=0.16$.
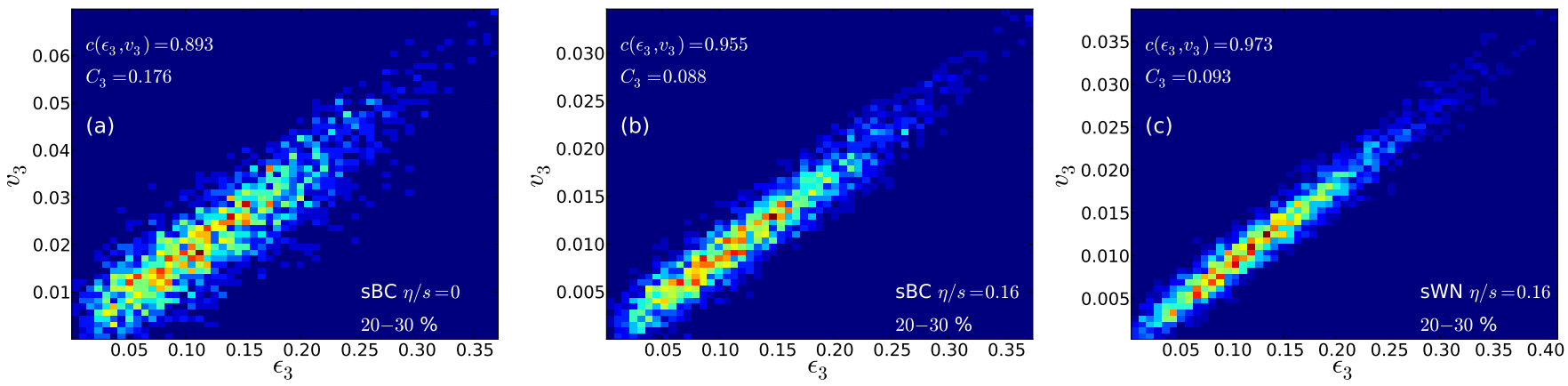

FIG. 2. $\epsilon_{3}$ and $v_{3}$ of pions in the $20-30 \%$ centrality class using different initializations and viscosities. a) $\mathrm{sBC}$ and $\eta / s=0$, b) $\mathrm{sBC}$ and $\eta / s=0.16$ and c) $\mathrm{sWN}$ and $\eta / s=0.16$.
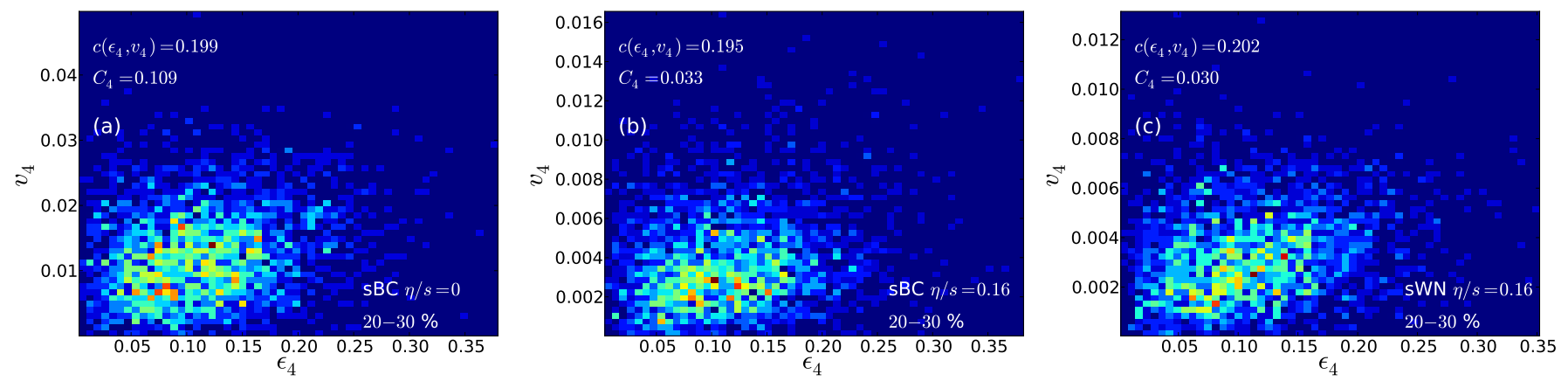

FIG. 3. $\epsilon_{4}$ and $v_{4}$ of pions in the $20-30 \%$ centrality class using different initializations and viscosities. a) $\mathrm{sBC}$ and $\eta / s=0$, b) $\mathrm{sBC}$ and $\eta / s=0.16$ and c) $\mathrm{sWN}$ and $\eta / s=0.16$.

As can be seen in these figures, the $v_{2}$ and $v_{3}$ coefficients display a strong linear correlation to their corresponding initial-state coefficients for all cases considered. This is confirmed by the values of the linear correlation coefficient $c\left(v_{2}, \epsilon_{2}\right) \sim c\left(v_{3}, \epsilon_{3}\right) \sim 1$, as shown in the Figures (top left corner). As for any two variables we can write

$$
v_{n}=C_{n} \epsilon_{n}+\delta_{n}
$$

where $C_{n}=\left\langle v_{n}\right\rangle_{\mathrm{ev}} /\left\langle\epsilon_{n}\right\rangle_{\mathrm{ev}}$, and consequently, $\left\langle\delta_{n}\right\rangle_{\mathrm{ev}}=0$. The values of $C_{n}$ are shown in Figs. 1 and 2 For $n=2$ a linear relation, $v_{2}=C_{2} \epsilon_{2}$, is approximately satisfied event-by-event with only $\sim 10 \%$ deviations from this relation at a given $\epsilon_{2}$. On the other hand, an event-by-event linear relation between $v_{3}$ and $\epsilon_{3}$ is not satisfied well, with $v_{3}$ deviating $\sim 50 \%$ from $v_{3}=C_{3} \epsilon_{3}$ at a given $\epsilon_{3}$.

In all the cases considered above, there is basically no linear correlation between $\epsilon_{4}$ and $v_{4}$, see Fig. 3 . At least one reason for this behavior is that there is also correlation between $\epsilon_{2}^{2}$ and $v_{4}$, which can be of the same order or larger than $c\left(\epsilon_{4}, v_{4}\right): c\left(\epsilon_{2}^{2}, v_{4}\right)=0.40(\mathrm{sBC}, \eta / s=0), c\left(\epsilon_{2}^{2}, v_{4}\right)=0.69(\mathrm{sBC}, \eta / s=0.16)$ and, $c\left(\epsilon_{2}^{2}, v_{4}\right)=0.46(\mathrm{sWN}$, 
$\eta / s=0.16)$. This is a non-linear effect triggered by the coupling between two different Fourier coefficients, i.e. $n=2$ and $n=4$, and a linear combination of these two components was found to be a good estimator for $v_{4}$ [21].

As expected, the proportionality coefficients $C_{n}$ are sensitive to the value of the shear viscosity. This can be seen by comparing Figs. 1 $\mathrm{a}$ and $1 \mathrm{~b}(\mathrm{n}=2)$, Figs. $2 \mathrm{a}$ and $2 \mathrm{~b}(\mathrm{n}=3)$, and Figs. $3 \mathrm{k}$ and $3 \mathrm{~b}(\mathrm{n}=4)$. In general, the higher Fourier coefficients are expected to be more sensitive to the viscosity [19]. This is also the case in our calculations, and is confirmed by comparing the relative changes in the coefficients $C_{2}, C_{3}$ and $C_{4}$.

Note that the proportionality constants $C_{n}$ do not depend only on the intrinsic properties of the fluid, but also on the initial conditions. Again something to be expected, since in the calculations done using averaged initial conditions, the precise value of the proportionality depended on many details as discussed in the Introduction.
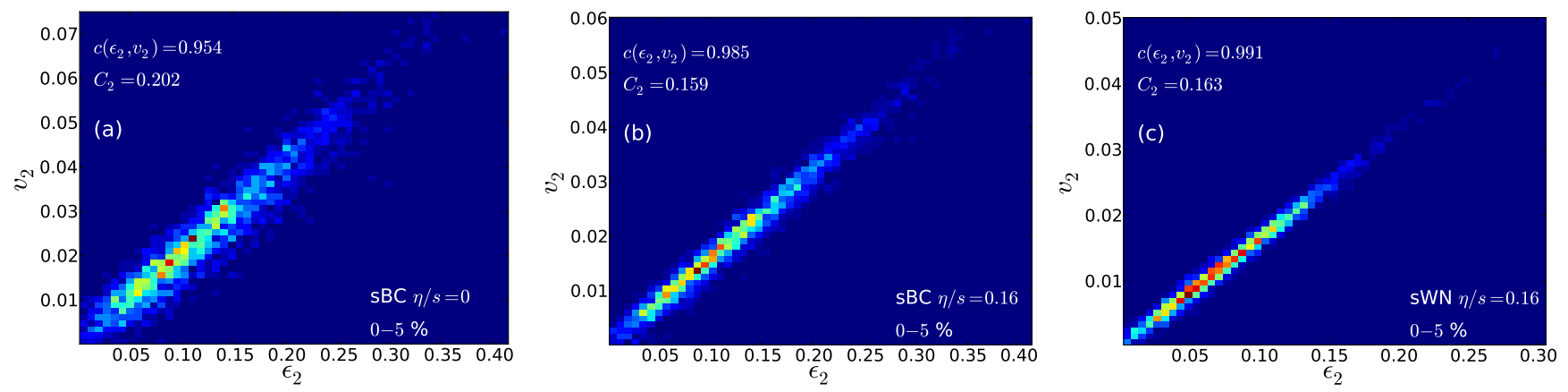

FIG. 4. $\epsilon_{2}$ and $v_{2}$ in the $0-5 \%$ centrality class using different initializations and viscosities. a) $\mathrm{sBC}$ and $\left.\eta / s=0, \mathrm{~b}\right) \mathrm{sBC}$ and $\eta / s=0.16$ and $\mathrm{c}$ ) sWN and $\eta / s=0.16$.
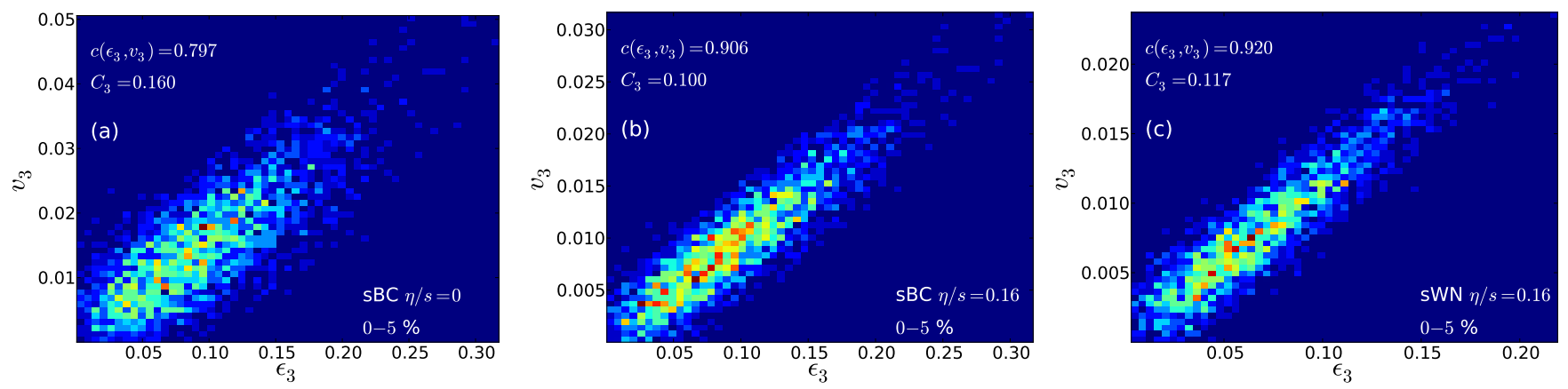

FIG. 5. $\epsilon_{3}$ and $v_{3}$ in the $0-5 \%$ centrality class using different initializations and viscosities. a) $\mathrm{sBC}$ and $\left.\eta / s=0, \mathrm{~b}\right) \mathrm{sBC}$ and $\eta / s=0.16$ and $\mathrm{c}$ ) $\mathrm{sWN}$ and $\eta / s=0.16$.
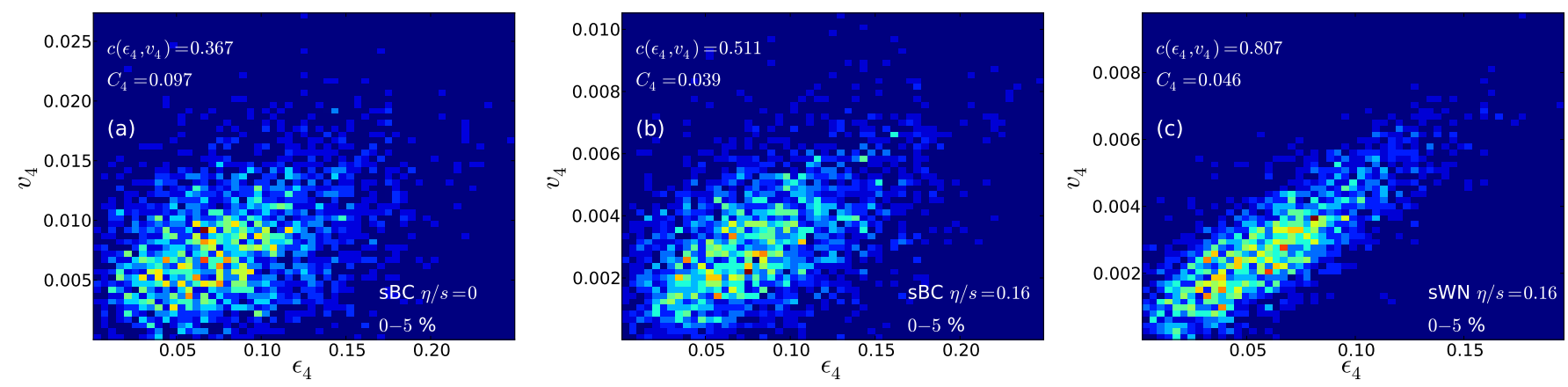

FIG. 6. $\epsilon_{4}$ and $v_{4}$ in the $0-5 \%$ centrality class using different initializations and viscosities. a) $\mathrm{sBC}$ and $\left.\eta / s=0, \mathrm{~b}\right) \mathrm{sBC}$ and $\eta / s=0.16$ and $\mathrm{c}) \mathrm{sWN}$ and $\eta / s=0.16$.

In Figs. 4 and 5 we show the 2-dimensional histograms of $\epsilon_{2}$ and $v_{2}$ and of $\epsilon_{3}$ and $v_{3}$, respectively, in the $0-5 \%$ centrality class. We plot the same cases considered above: (a) $\mathrm{sBC}$ initialization with $\eta / s=0$, (b) $\mathrm{sBC}$ initialization 
with $\eta / s=0.16$, and (c) sWN initialization with $\eta / s=0.16$. For $n=2$ and $n=3$ the linear correlation is still valid. Also, the effect of shear viscosity and initialization on $C_{n}$ remain qualitatively the same. On the other hand, in Fig. 6 the correlation between $\epsilon_{4}$ and $v_{4}$ in central collisions is drastically different from the correlation in the $20-30 \%$ centrality class. In the $0-5 \%$ centrality class the linear correlation coefficient $c\left(\epsilon_{4}, v_{4}\right)$ becomes much closer to 1 when compared to the peripheral case. It can be as large as $\sim 0.81$ obtained for the sWN initialization with $\eta / s=0.16$. This behavior is expected since in Ref. 21] it was shown that $\epsilon_{4}$ becomes a better estimator for $v_{4}$ in central collisions.

\section{B. distributions of $v_{n}$}

So far the event-averaged values of $v_{n}$ have been extensively studied. In order to observe what can be learned by looking at $v_{n}$ probability distributions, it is convenient to remove the average from the distributions, and study the relative fluctuations using the scaled variables

$$
\delta v_{n}=\frac{v_{n}-\left\langle v_{n}\right\rangle_{\mathrm{ev}}}{\left\langle v_{n}\right\rangle_{\mathrm{ev}}}, \quad \text { and } \quad \delta \epsilon_{n}=\frac{\epsilon_{n}-\left\langle\epsilon_{n}\right\rangle_{\mathrm{ev}}}{\left\langle\epsilon_{n}\right\rangle_{\mathrm{ev}}}
$$

In this way changes in the probability distributions due to changes in the average values are removed.

It was shown in the previous subsection that $v_{n}$ and $\epsilon_{n}$ have a strong linear correlation for $n=2$ and 3 . As discussed in the Appendix, if two variables are linearly correlated, and $\langle d\rangle=0$, the variances of the relative distributions are equal. Since viscosity has only a small effect on the correlations of $v_{n}$ and $\epsilon_{n}$, we expect that $\sigma_{v_{n}}^{2} \approx \sigma_{\epsilon_{n}}^{2}$, independent of viscosity. In such a case the information about the fluid response to the initial geometry is contained in the coefficients $C_{n}$ controlling the average $\left\langle v_{n}\right\rangle_{\mathrm{ev}}$, while the relative fluctuations of $v_{n}$ originate from the relative fluctuations of $\epsilon_{n}$ and do not depend on viscosity of the fluid.
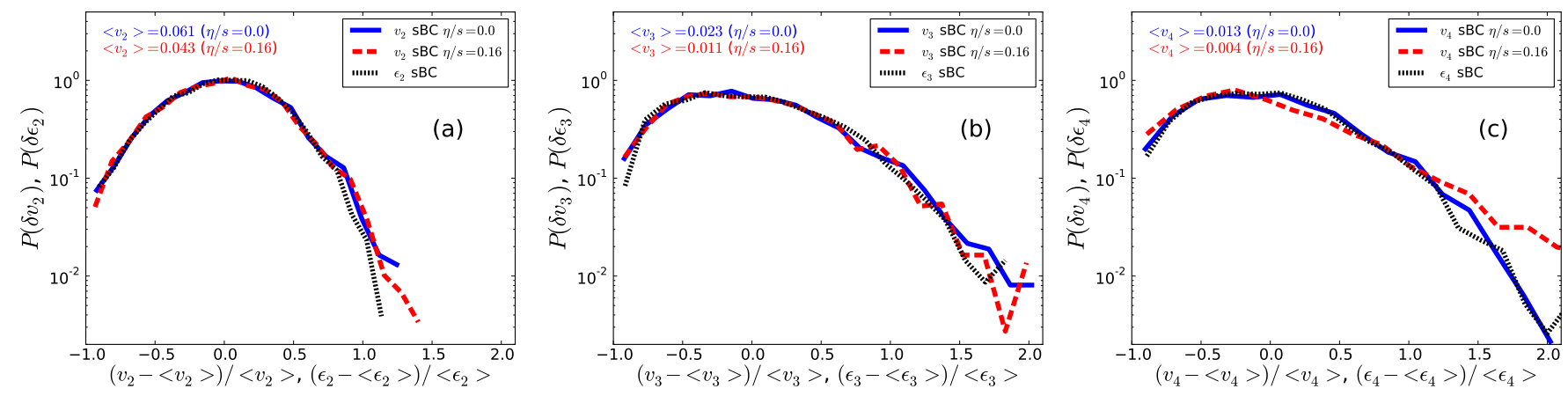

FIG. 7. Probability distributions: a) $P\left(\delta v_{2}\right)$ and $P\left(\delta \epsilon_{2}\right)$, b) $P\left(\delta v_{3}\right)$ and $P\left(\delta \epsilon_{3}\right)$, and c) $P\left(\delta v_{4}\right)$ and $P\left(\delta \epsilon_{4}\right)$, in the $20-30 \%$ centrality class with $\mathrm{sBC}$ initialization and two different values of $\eta / s, \eta / s=0$ and $\eta / s=0.16$.
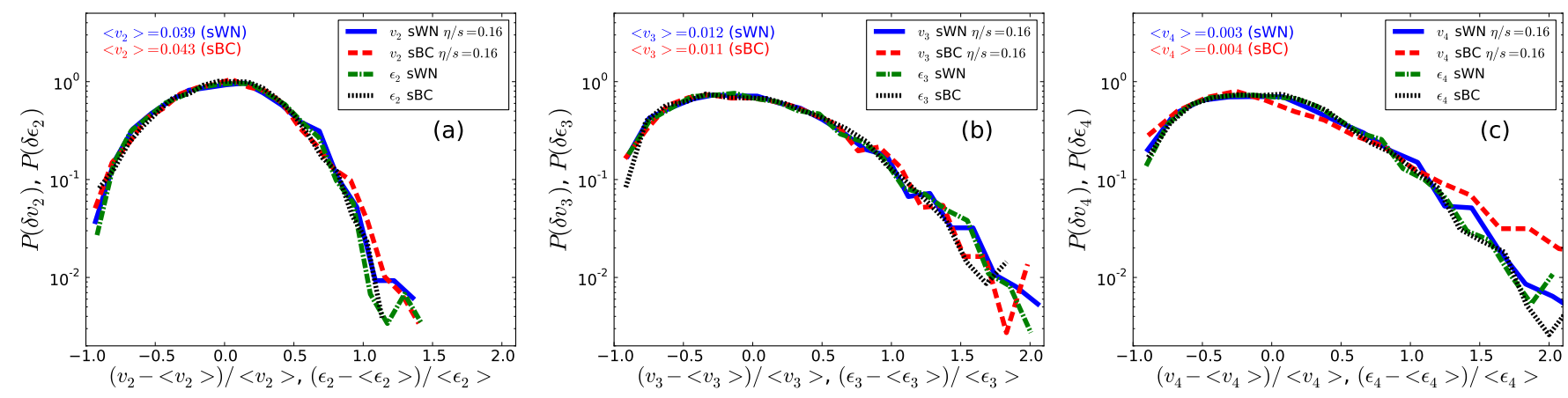

FIG. 8. Probability distributions: a) $P\left(\delta v_{2}\right)$ and $P\left(\delta \epsilon_{2}\right)$, b) $P\left(\delta v_{3}\right)$ and $P\left(\delta \epsilon_{3}\right)$, and c) $P\left(\delta v_{4}\right)$ and $P\left(\delta \epsilon_{4}\right)$, in the $20-30 \%$ centrality class with $\eta / s=0.16$ and two different initial conditions, $\mathrm{sBC}$ and sWN.

To test this assumption, and to see whether similarity extends beyond variances, we plot the probability distributions $P\left(\delta v_{n}\right)$ and $P\left(\delta \epsilon_{n}\right)$ in $20-30 \%$ centrality class in Fig. 7 using the sBC initialization and two values of viscosity, 
$\eta / s=0$ and 0.16. As seen in panel a) for $n=2$ and in panel b) for $n=3$, not only the variances are similar, but the entire distributions are almost identical. Fig. $7 \mathrm{c}$ depicts the relative distributions for $n=4$, and surprisingly they are very similar even if $v_{4}$ and $\epsilon_{4}$ are not linearly correlated. There are deviations only at the tail of the distribution. As discussed in the Appendix, lack of correlation leads to a large spread of possible values of $v_{4}$ at given $\epsilon_{4}$ which tends to make $\delta v_{4}$ distribution wider, but this effect can be canceled by other terms. How these terms arise is an interesting question beyond the scope of this paper.

To check whether the similarity of $P\left(\delta v_{n}\right)$ and $P\left(\delta \epsilon_{n}\right)$ is only a coincidence based on the sBC initialization, we show in Fig. 8 the distributions using both sBC and sWN initialization, but using only one value of viscosity, $\eta / s=0.16$. Again, panels a, b, and c depict cases with $n=2,3$, and 4 , respectively. $P\left(\delta v_{n}\right)$ and $P\left(\delta \epsilon_{n}\right)$ are almost equal for both initializations. The distributions $P\left(\delta \epsilon_{n}\right)$ are also similar for both initializations, but this is because both Glauber-type initializations give rise to the same relative fluctuations of initial state anisotropies, see discussion in Ref. [22].

These results are valid in the $0-5 \%$ centrality class as well. We have also checked that $P\left(\delta v_{n}\right)$ is not sensitive to the freeze-out temperature within interval $100<T_{\text {fo }}<160 \mathrm{MeV}$. Thus the distribution of relative fluctuations of $v_{n}$ could be the ideal observable to study the fluctuations of the initial geometry. If fluid dynamics provides a correct description of heavy-ion collisions, $P\left(\delta v_{n}\right)$ is a direct measurement of the initial state anisotropy fluctuations and can be compared directly to initial condition models, such as Monte-Carlo Glauber or various implementations of Color-Glass Condensate based initial conditions [23].

\section{C. $\left(v_{n}, v_{m}\right)$ linear correlations}
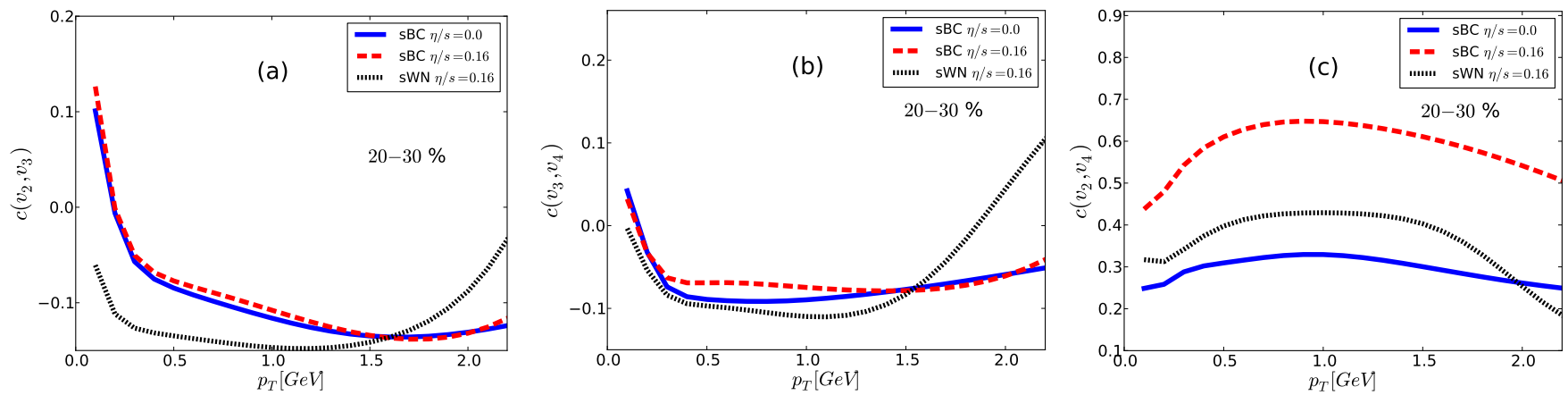

FIG. 9. Correlations: a) $c\left(v_{2}, v_{3}\right)$, b) $c\left(v_{3}, v_{4}\right)$, and c) $c\left(v_{2}, v_{4}\right)$, as function of transverse momentum in the $20-30 \%$ centrality class using different initializations and viscosities.

\begin{tabular}{|c|c|c|c|c|c|c|}
\hline & $c\left(\epsilon_{2}, \epsilon_{3}\right)$ & $c\left(v_{2}, v_{3}\right)$ & $c\left(\epsilon_{2}, \epsilon_{4}\right)$ & $c\left(v_{2}, v_{4}\right)$ & $c\left(\epsilon_{3}, \epsilon_{4}\right)$ & $c\left(v_{3}, v_{4}\right)$ \\
\hline $\mathrm{sBC} \eta / s=0.0$ & -0.09 & -0.11 & 0.26 & 0.32 & -0.03 & -0.11 \\
\hline $\mathrm{sBC} \eta / s=0.16$ & -0.09 & -0.11 & 0.25 & 0.63 & -0.03 & -0.09 \\
\hline $\mathrm{sWN} \eta / s=0.16$ & -0.15 & -0.14 & -0.04 & 0.42 & 0.03 & -0.11 \\
\hline
\end{tabular}

TABLE I. Linear correlation coefficients for $p_{T}$-integrated $v_{n}$ 's and $\epsilon_{n}$ 's in the $20-30 \%$ centrality class.

We computed the linear correlation coefficients, $c\left(v_{2}, v_{3}\right), c\left(v_{2}, v_{4}\right)$, and $c\left(v_{3}, v_{4}\right)$ as a function of the transverse momentum, $p_{T}$. We found that $c\left(v_{2}, v_{3}\right) \sim c\left(v_{3}, v_{4}\right) \sim 0$ and, therefore, are not linearly correlated. These correlations are shown in Fig. 9] and 9]. We also show the values of the correlation coefficient between the integrated $v_{n}$ 's and of the coefficients $\epsilon_{n}$ in Table I. It should be noted that, even though both Glauber initializations used in this paper have the same relative anisotropy fluctuations, their correlations differ.

Figure 9r shows the correlation coefficient between $v_{2}$ and $v_{4}$ as a function of $p_{T}$. As can be read off from the Figure, $c\left(v_{2}, v_{4}\right)$ depends strongly on $\eta / s$ and, consequently, is sensitive to the properties of the QGP. Also, this correlation function is strongly affected by the correlation between $\epsilon_{2}$ and $\epsilon_{4}$ existing in the initial state, i.e., initial conditions with different $c\left(\epsilon_{2}, \epsilon_{4}\right)$ lead to very different $c\left(v_{2}, v_{4}\right)$, see the dashed and dotted lines in Fig. 95. Overall, just like $v_{2}$ probes the fluid-dynamical response to an initial geometry (characterized by $\left.\epsilon_{2}\right), c\left(v_{2}, v_{4}\right)$ probes the fluid-dynamical response to correlations in the initial geometry (in this case characterized by $c\left(\epsilon_{2}, \epsilon_{4}\right)$ ).

In our investigations, $c\left(v_{2}, v_{4}\right)$ is the only correlation that was sensitive to both the fluctuations of the initial condition and the transport properties of the fluid. This correlation can thus be used as a further constraint to the 
fluid-dynamical models applied to heavy-ion collisions.

\section{CONCLUSIONS}

In this paper, we studied the relation between $v_{n}$ and $\epsilon_{n}$ in ultrarelativistic heavy-ion collisions using event-by-event fluid dynamics. We confirmed that the second and third Fourier coefficients have a strong linear correlation to the initial geometry of the collision. We showed that while the event-average Fourier coefficients, $\left\langle v_{n}\right\rangle_{\mathrm{ev}}, n=2,3$, and 4 , are sensitive to the details of the fluid-dynamical evolution, their relative fluctuations, $\delta v_{n}=\left(v_{n}-\left\langle v_{n}\right\rangle_{\mathrm{ev}}\right) /\left\langle v_{n}\right\rangle_{\mathrm{ev}}$, are determined solely by the fluctuations of the corresponding initial state anisotropy coefficients, with basically no sensitivity to the viscosity of the fluid. This makes the distribution of $\delta v_{n}$ a direct probe of the initial condition of a heavy-ion collision, providing a direct and clean measurement of the distribution of the relative fluctuations of the initial anisotropy, i.e.,

$$
P\left(\delta v_{n}\right) \simeq P\left(\delta \epsilon_{n}\right), \quad n=2,3,4 .
$$

Surprisingly, this relation was shown to be true even for the relative fluctuations of $v_{4}$, even though $v_{4}$ itself is not linearly correlated to $\epsilon_{4}$.

Furthermore we found that the linear correlations between the flow harmonics $v_{n}$ are not solely dominated by the initial conditions. Especially the correlation function $c\left(v_{2}, v_{4}\right)$ is sensitive to the viscosity of the fluid providing an additional constraint for the model when one tries to extract the viscosity coefficient from the data.

\section{ACKNOWLEDGEMENTS}

The authors thank D.H. Rischke and J. Jia for discussions. The work of H.N. was supported by Academy of Finland, Project No. 133005, G.S.D. by Helmholz Research School H-QM, H.H. by the ExtreMe Matter Institute (EMMI), and P.H. by BMBF under contract no. 06FY9092. We acknowledge CSC - IT Center for Science in Espoo, Finland, for the allocation of computational resources.

\section{Appendix: Relative widths of correlated distributions}

A relation between variables $\epsilon$ and $v$ (we omit the indexes $n$ here) can be written as

$$
v=k \epsilon+d .
$$

If we define

$$
k=\frac{\sigma_{v}}{\sigma_{\epsilon}} c(\epsilon, v),
$$

where $c(\epsilon, v)$ is the linear correlator (Eq. (잠), $\epsilon$ and $d$ are not linearly correlated, $c(\epsilon, d)=0$. It also follows that

$$
\sigma_{d}^{2}=\sigma_{v}^{2}\left(1-c(\epsilon, v)^{2}\right),
$$

i.e. the stronger the linear (anti)correlation between $\epsilon$ and $d$, the narrower the distribution of $d$.

For the distributions of the scaled variables, $\delta x=(x-\langle x\rangle) /\langle x\rangle$, it holds that $\sigma_{\delta x}=\sigma_{x} /\langle x\rangle$, and that $\langle\delta x\rangle=0$. The variance of $\delta v$ can now be written as

$$
\begin{aligned}
\sigma_{\delta v}^{2} & =\frac{1}{\langle v\rangle^{2}}\left[k^{2} \sigma_{\epsilon}^{2}+2 k \sigma_{\epsilon} \sigma_{d} c(\epsilon, d)+\sigma_{d}^{2}\right] \\
& =\frac{\sigma_{\epsilon}^{2}}{\langle\epsilon\rangle^{2}}\left[\frac{\langle\epsilon\rangle^{2}}{\langle v\rangle^{2}} k^{2}+\frac{\langle\epsilon\rangle^{2}}{\langle v\rangle^{2}} \frac{\sigma_{d}^{2}}{\sigma_{\epsilon}^{2}}\right] \\
& =\sigma_{\delta \epsilon}^{2}\left[\frac{1}{\left(1+\frac{\langle d\rangle}{k\langle\epsilon\rangle}\right)^{2}}+\frac{\langle\epsilon\rangle^{2}}{\langle v\rangle^{2}} \frac{\sigma_{d}^{2}}{\sigma_{\epsilon}^{2}}\right] .
\end{aligned}
$$

As mentioned, if $c(\epsilon, v) \approx 1, \sigma_{d} \approx 0$, and the last term in Eq. A.4 is negligible. If also $\langle d\rangle \approx 0, \sigma_{\delta v} \approx \sigma_{\delta \epsilon}$. Roughly speaking the requirement $\langle d\rangle \approx 0$ means that when $\epsilon \rightarrow 0,\langle v\rangle \approx 0$-a requirement our distributions with $n=2$ 
and 3 fulfill as seen in Figs. 1, 2, 4, and 5, On the other hand, the correlation is weak for $n=4$ in the $20-30 \%$ centrality class. In that case the $d$ distribution is wide, but $\langle d\rangle \neq 0$ as well, and the two terms in Eq. (A.4) sum to approximately one, and the distributions of $\delta \epsilon$ and $\delta v$ are equal even in that case.

[1] M. Gyulassy and L. McLerran, Nucl. Phys. A 750, 30 (2005).

[2] P. F. Kolb and U. W. Heinz, In *Hwa, R.C. (ed.) et al.: Quark gluon plasma 3* 634-714 [nucl-th/0305084].

[3] H. Song and U. W. Heinz, Phys. Rev. C 78, 024902 (2008) [arXiv:0805.1756 [nucl-th]].

[4] P. Huovinen, In *Hwa, R.C. (ed.) et al.: Quark gluon plasma 3* 600-633 [nucl-th/0305064].

[5] C. E. Aguiar, Y. Hama, T. Kodama and T. Osada, Nucl. Phys. A 698, 639 (2002); O. Socolowski, Jr., F. Grassi, Y. Hama and T. Kodama, Phys. Rev. Lett. 93, 182301 (2004); R. Andrade, F. Grassi, Y. Hama, T. Kodama and O. Socolowski, Jr., Phys. Rev. Lett. 97, 202302 (2006); J. Takahashi, B. M. Tavares, W. L. Qian, R. Andrade, F. Grassi, Y. Hama, T. Kodama and N. Xu, Phys. Rev. Lett. 103, 242301 (2009).

6] B. Alver and G. Roland, Phys. Rev. C 81, 054905 (2010) [Erratum-ibid. C 82, 039903 (2010)] .

[7] B. Schenke, S. Jeon and C. Gale, Phys. Rev. Lett. 106, 042301 (2011); B. Schenke, S. Jeon and C. Gale, Phys. Lett. B 702, 59 (2011); B. Schenke, S. Jeon and C. Gale, Phys. Rev. C 85, 024901 (2012); Z. Qiu and U. W. Heinz, Phys. Lett. B 717, 261 (2012); Z. Qiu, C. Shen and U. Heinz, Phys. Lett. B 707, 151 (2012); H. Petersen, G. -Y. Qin, S. A. Bass and B. Muller, Phys. Rev. C 82, 041901 (2010); H. Petersen, V. Bhattacharya, S. A. Bass and C. Greiner, Phys. Rev. C 84, 054908 (2011); H. Petersen, Phys. Rev. C 84, 034912 (2011); P. Bozek and W. Broniowski, Phys. Rev. C 85, 044910 (2012); P. Bozek and W. Broniowski, Phys. Rev. Lett. 109, 062301 (2012); P. Bozek, Phys. Lett. B 717, 287 (2012); D. Teaney and L. Yan, Phys. Rev. C 86, 044908 (2012); J. Jia and D. Teaney, arXiv:1205.3585 [nucl-ex]; D. Teaney and L. Yan, Phys. Rev. C 83, 064904 (2011).

[8] H. Holopainen, H. Niemi and K. J. Eskola, Phys. Rev. C 83, 034901 (2011); H. Holopainen, H. Niemi and K. J. Eskola, Nucl. Phys. A 855, 486 (2011); H. Holopainen, H. Niemi and K. J. Eskola, J. Phys. G G 38, 124164 (2011).

[9] J. Jia [ATLAS Collaboration], arXiv:1209.4232 [nucl-ex].

[10] C. Gale, S. Jeon, B. Schenke, P. Tribedy and R. Venugopalan, arXiv:1209.6330 [nucl-th].

[11] M. Alvioli, H. Holopainen, K. J. Eskola and M. Strikman, Phys. Rev. C 85, 034902 (2012); M. Alvioli, H. Holopainen, K. J. Eskola and M. Strikman, PoS QNP 2012, 172 (2012).

[12] H. Niemi, G. S. Denicol, P. Huovinen, E. Molnar and D. H. Rischke, Phys. Rev. C 86, 014909 (2012); H. Niemi, G. S. Denicol, P. Huovinen, E. Molnar and D. H. Rischke, Phys. Rev. Lett. 106, 212302 (2011).

[13] P. Huovinen and P. Petreczky, Nucl. Phys. A 837, 26 (2010).

[14] W. Israel and J. M. Stewart, Phys. Lett. 58A, 213 (1976); Ann. Phys. (N.Y.) 118, 341 (1979); Proc. Roy. Soc. London A 365, 43 (1979).

[15] G. S. Denicol, H. Niemi, E. Molnar, and D. H. Rischke, Phys. Rev. D 85, 114047 (2012).

[16] G. S. Denicol, T. Koide, and D. H. Rischke, Phys. Rev. Lett. 105, 162501 (2010).

[17] E. Molnar, H. Niemi, and D. H. Rischke, Eur. Phys. J. C 65, 615 (2010).

[18] F. Cooper and G. Frye, Phys. Rev. D 10, 186 (1974).

[19] B. H. Alver, C. Gombeaud, M. Luzum and J. -Y. Ollitrault, Phys. Rev. C 82, 034913 (2010).

[20] Z. Qiu and U. W. Heinz, Phys. Rev. C 84, 024911 (2011).

[21] F. G. Gardim, F. Grassi, M. Luzum and J. -Y. Ollitrault, Phys. Rev. C 85, 024908 (2012).

[22] W. Broniowski, P. Bozek and M. Rybczynski, Phys. Rev. C 76, 054905 (2007) [arXiv:0706.4266 [nucl-th]].

[23] A. Adil, H. -J. Drescher, A. Dumitru, A. Hayashigaki, and Y. Nara, Phys. Rev. C 74, 044905 (2006); H.-J. Drescher and Y. Nara, Phys. Rev. C 75, 034905 (2007); H.-J. Drescher and Y. Nara, Phys. Rev. C 76, 041903 (2007); Y Nara, Prog. Theor. Phys. Suppl. 193, 145 (2012); A. Dumitru and Y. Nara, Phys. Rev. C 85, 034907 (2012); B. Schenke, P. Tribedy, and R. Venugopalan, Phys. Rev. Lett. 108, 252301 (2012). 